\title{
The Utility of Latent Class Analysis to Understand Heterogeneity in Youth Coping Strategies: A Methodological Introduction
}

Behavioral Disorders

I-I5

(C) Hammill Institute on Disabilities 2022

Article reuse guidelines:

sagepub.com/journals-permissions DOI: 10.1 | 77/019874292| |0672|4

journals.sagepub.com/home/bhd

(9)AGE

\author{
Karen Nylund-Gibson, PhD' (iD, Adam C. Garber, MA', Jay Singh, BS', \\ Melissa R. Witkow, PhD ${ }^{2}$, Adrienne Nishina, PhD $^{3}$, and Amy Bellmore, PhD $^{4}$
}

\begin{abstract}
Latent class analysis (LCA) is a useful statistical approach for understanding heterogeneity in a population. This article provides a pedagogical introduction to LCA modeling and provides an example of its use to understand youth's daily coping strategies. The analytic procedures are outlined for choosing the number of classes and integration of the LCA variable within a structural equation model framework, specifically a latent class moderation model, and a detailed table provides a summary of relevant modeling steps. This applied example demonstrates the modeling context when the LCA variable is moderating the association between a covariate and two outcome variables. Results indicate that students' coping strategies moderate the association between social stress and negative mood; however, they do not moderate the social stress-positive mood association. Supplemental Material include R (MplusAutomation) code to automate the enumeration procedure, ML three-step auxiliary variable integration, and the generation of figures for visually depicting LCA results.
\end{abstract}

\section{Keywords}

latent class analysis (LCA), moderation, student coping, auxiliary variables, mixture modeling, structural equation modeling

Latent class analysis (LCA) is becoming a widely used statistical technique for a range of social scientists. This analytic approach allows researchers to address questions that involve grouping individuals based on who respond similarly to a set of observed items. This modeling approach provides a way for researchers to better understand patterns of responses to a set of observed measures, allowing a deeper and more parsimonious understanding of unmeasured groups or types within the data that alternative modeling approaches are not able to reveal.

Applications of LCA are found across a wide range of research fields and have been widely used within the social sciences. For example, LCA has been used in epidemiology to assess the effectiveness of a bullying and victimization prevention program (Tsiantis et al., 2013) and in behavioral disorder research to investigate the correspondence between teacher management practices and elementary school students' disruptive behavior (Gage et al., 2018), among other applications. A recent study by Kavčič et al. (2021), aimed at understanding coping strategies during the COVID 19 pandemic, found three coping strategy profiles that aligned with previous work in which LCA was used to investigate coping. This study found that individuals in the engaged coping profile group had the best mental health outcomes.
Common among these fields is a trend toward valuing modeling approaches that are capable of revealing heterogeneity within populations and go beyond descriptions of samples based on standard regression-type estimates (i.e., unconditional means).

\section{Empirical Creation of Groups}

The general appeal of LCA is that it is a statistical method that can be used to create groups, or latent classes, to describe a population. These latent classes are thought of as unobserved "subgroups" or "typologies" that capture heterogeneity in a population with respect to a specific set of measured items. That is, LCA uses patterns in the set of observed items to identify groups of individuals who have

\footnotetext{
'University of California, Santa Barbara, USA

${ }^{2}$ Willamette University, Salem, OR, USA

${ }^{3}$ University of California, Davis, USA

${ }^{4}$ Univerity of Wisconsin-Madison, USA

Corresponding Author:

Karen Nylund-Gibson, University of California Santa Barbara, Santa Barbara, CA 93106, USA.

Email: knylund@education.ucsb.edu
} 
similar response patterns. Thus, LCA is a multivariate approach to creating classifications, which is in contrast to creating groups using cutoff scores that impose arbitrary boundary conditions to determine class membership (Nylund, Bellmore, et al., 2007). Once classes are identified, associations with other variables can be made to better understand who comprises these latent groups as well as associations with how class membership relates to a set of distal outcomes. The procedure of conducting an LCA with covariates and distal outcomes is a multistep process (Nylund-Gibson et al., 2019) that can be overwhelming to a researcher attempting this approach for the first time. To provide a roadmap for researchers to follow, we have included a table with details of the modeling steps which can be found in Supplemental Material.

The use of cutoff scores to create groups can be a problematic procedure that can lead to bias, misclassification, and models that overlook meaningful nuances of complex processes (Leon-Perez et al., 2014). When creating cutoff scores, a concern has to do with the often arbitrary nature of the placement of the cut point. This introduces a problem of misclassification of individuals who are close to the cutoff. As a hypothetical example, if it is decided that the cutoff threshold for being labeled "depressed" is the endorsement of nine or more of 12 items, individuals who endorse eight items are then classified as "not depressed" although they only had one fewer depression symptom endorsed. Furthermore, there is the risk of setting a threshold precedent that future researchers will follow that lacks adequate empirical justification (Dwyer, 1996). Often it is the case that individuals do not fit perfectly into these created groups but rather are classified with some degree of classification error; this classification uncertainty is a key feature of the LCA model.

Furthermore, creating groups using a single scale or item ignores the possibility that individuals vary on a range of dimensions simultaneously making groupings based on a univariate model unsatisfactory. Consider, for example, a school counselor who is interested in supporting the development of positive coping strategies among students. She may want to understand the collective needs across multiple domains and, as a result, may use more than one piece of information to decide who needs an intervention. One single indicator of a student's coping strategy may be insufficient, but observations across multiple contexts could provide a more holistic view of which students are most in need of intervention (e.g., classes or groups). LCA provides a modeling context in which classification is derived on a set of indicators, not just a single indicator.

In social science research, the creation of composite scores in the form of total scores or mean scores is common. This practice has the consequence of blurring or canceling out nuances in response patterns that may be both interesting and meaningful (Little et al., 2002). For example, two observations may have the same mean or total score due to the summation across different combinations of items, each possibly having a different meaning. Implied is that each item is equally weighted in the creation of the score, which may not be reflective of the theory driving the measurement of the construct. In our applied example, some coping items are adaptive (e.g., talked to someone) and others are less adaptive (e.g., got mad) so simply adding them together and creating a total or mean score could hide meaningful variation.

There are a range of other classification techniques that exist such as cluster analysis or $k$-means clustering. Although these methods provide a way for classifying individuals on a set of observed indicators, an important disadvantage to these approaches is that they are not model-based, and therefore fit statistics such as information criteria cannot be used for evaluation (Magidson \& Vermunt, 2002). LCA is a model-based approach to classification and permits a statistical evaluation of how well a proposed LCA model represents the data. In addition, as the emergent groups in LCA are based on a model, these latent classes may be tested using replication procedures (Whittaker \& Miller, 2021). In practice, the $k$-means clustering and LCA solutions may be similar (Brusco et al., 2017), meaning that a comparison of how individuals are put into groups may have high congruence. Although solutions may be similar, the mixture modeling approach allows for statistical evaluation of fit and allows for the integration of the mixture model within a larger structural equation modeling (SEM) context (e.g., with covariate and distal outcomes). The advantages of mixture modeling relative to $k$-means clustering are outlined in Vermunt (2011).

\section{The Aim of the Present Article}

Although there are methodological and substantive articles on the use of LCA, this article aims to provide an overview of LCA by demonstrating its application in the context of youth coping strategies. The article will provide an overview of the statistical model, the modeling procedures with a detailed table of the phases of research (see Table S1 of Supplemental Material), and demonstrate using an example of students' coping strategies. Next, we embed the latent class variable into a moderation model, showing how the emergent latent variable can be used as a moderator. We provide software syntax for the estimation of the models using Mplus (Muthén \& Muthén, 2017) through the R package MplusAutomation (Hallquist \& Wiley, 2018), which is used to automate the LCA enumeration process and threestep approach (see Supplemental Material).

\section{Applied Example: Patterns in Students' Coping}

To demonstrate the use of LCA, we investigate high school students' use of coping strategies commonly used in different situations (Wang \& Yip, 2020). Table 1 presents the 
Table I. Descriptive Statistics for Variables Used in the Coping LCA Example Including Indicator Variables (Five Coping Items), Covariates (Social Stress), and the Distal Outcomes (Positive and Negative Mood).

\begin{tabular}{|c|c|c|c|}
\hline Variables & $N$ & M & $S D$ \\
\hline \multicolumn{4}{|l|}{ Coping items (measurement model) } \\
\hline (I) I talked to somebody (e.g., friend, teacher, family member) about it. & 446 & 0.49 & 0.50 \\
\hline (2) I got mad and threw or hit something or cursed out loud to let off steam. & 446 & 0.09 & 0.28 \\
\hline (3) I did something to take my mind off of it, forgot the whole thing, or told myself it doesn't matter. & 444 & 0.52 & 0.50 \\
\hline (4) I thought about why it happened or how to prevent it in the future. & 445 & 0.52 & 0.50 \\
\hline (5) I felt sorry for myself, worried, or cried about it. & 444 & 0.23 & 0.42 \\
\hline \multicolumn{4}{|l|}{ Covariate and distal outcome variables } \\
\hline (X) Social Stress: Did this stressful situation involve other student(s)? & 442 & 0.71 & 0.45 \\
\hline$\left(Y_{1}\right)$ Positive Mood: Mean Score of items; Proud, Helpful to others, Respected, Sure about myself, Feels ok & 446 & 2.99 & 0.64 \\
\hline$\left(Y_{2}\right)$ Negative Mood: Mean Score of items; Scared, Nervous Tense, Mad at myself, Unsure about myself & 446 & 1.79 & 0.70 \\
\hline
\end{tabular}

wording of the five coping strategy items that compose the measurement portion of the model, along with the mean and standard deviations for each item from a sample of high school students. Students were asked to recall the most significant thing that had happened to them earlier in the day in school and then indicate if they used each coping strategy listed. For example, they reported "Yes" or "No" to the coping question, "I got mad and threw or hit something or cursed out loud to let off steam." Collectively, the items for the LCA are the students' responses to the five coping items.

LCA is a modeling technique that uses a set of responses, or the vector of observed responses, to create groups. One way to understand the utility of LCA is to look at the frequency of each possible pattern and how LCA collapses these patterns (see Table S1 in Supplemental Material). In our applied example, this would result in 32 possible patterns (i.e., five binary items with two response options, $2^{5}=32$ ). For any given example, some patterns may not be observed in the data set. Some patterns will not occur in a specific sample or may represent implausible combinations of responses, resulting in a reduced set of observed response patterns. While each observed pattern provides insight, however, it is generally hard to meaningfully make sense of such a large set of response patterns. Furthermore, with more items, the number of patterns increases exponentially, making interpretation intractable. LCA provides a tool to distill the most representative response patterns in a given sample, providing an interpretable solution for creating meaningful groups. In the coping example, LCA will identify groups of students who are similar with respect to their pattern of coping strategy use. That is, rather than studying all observed response patterns, we can create a manageable subset of patterns that more succinctly summarizes the variability among these patterns. It is important to note that by reducing the patterns we are inherently losing specificity; however, the rationale for the use of LCA, and mixtures in general, is that it provides a useful, parsimonious, and meaningful way to summarize patterns that otherwise would be difficult to describe.

For the sake of understanding, we present the emergent latent class solution for this example first, before introducing the statistical model so that when discussing statistical model parameters, we can refer to specific values from our example. After discussing the statistical parameters of the LCA model, we will describe the process of selecting a final LCA model. Unconventionally, we will present modeling results alongside the description of methodological procedures in this article, with the hope that the applied example will make the discussion of the statistical model less abstract for pedagogical purposes.

Coping example: 3-class solution. After running LCA with the five coping items, we choose the 3-class model as our final solution but also present the 2-class model for comparison as it was a viable alternative solution. Deciding on the number of classes is an important part of the process when conducting LCA, which is outlined later in this article. In LCA, it is conventional to present results in an item probability plot, as in Figure 1, which provides a way to interpret and label the emergent latent classes. Using estimated parameters found in the output, these plots present the information that is used to provide substantive labels to the emergent latent classes. The classes are arbitrarily ordered (i.e., 1, 2, 3 ), and it is up to the researcher to interpret the meaning of these classes using the conditional item probabilities. Like using loadings in factor analysis to label a factor, the conditional item probabilities (discussed in detail later) are used to interpret the classes, where items that most distinguish classes are often used in the naming of the emergent classes.

Looking at the lower plot in Figure 1, the three lines represent the conditional item probability profiles of three different latent classes. Across the $x$-axis are the five coping items while the $y$-axis represents the probability of endorsing a given item. Looking at the top dark solid line with circles, we see that this class is characterized by the high 


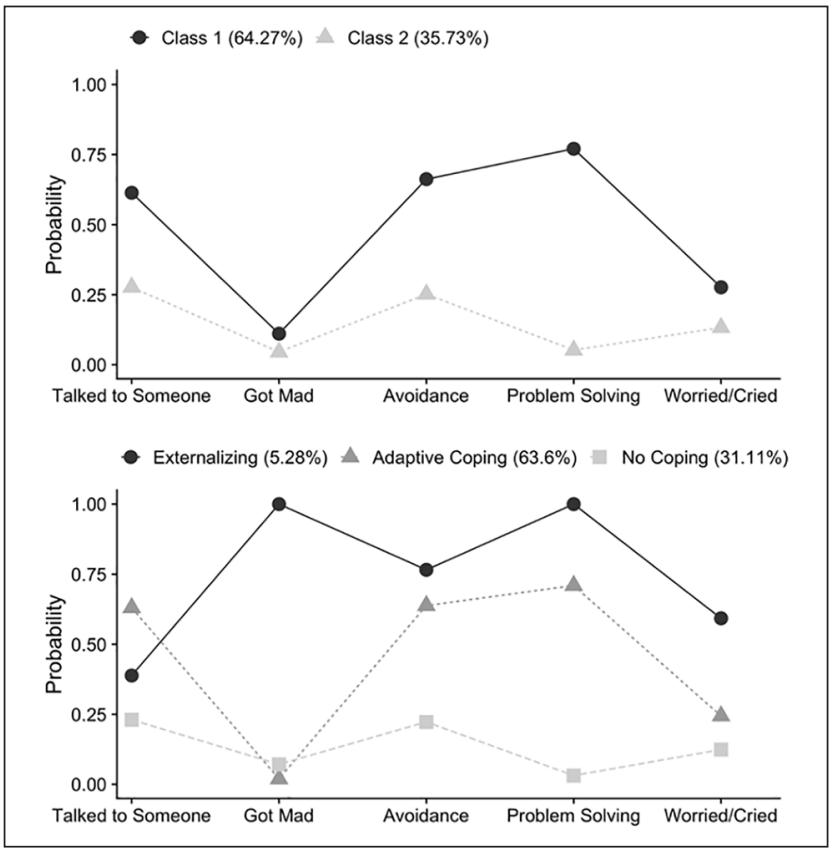

Figure I. Conditional item probability plots presenting the 2-class and 3-class LCA models of behavioral coping.

endorsement of the coping strategies "Got mad," "Avoidance," "Problem Solving," and moderate endorsement of the coping strategies "Talked to Someone," Worried/Cried." Because of this, we chose to label this class the Externalizing Behavior class, which exhibits coping with externalizing behavior. The externalizing coping item also distinguished this class from the other two classes. The Externalizing Behavior class was estimated to be $5.2 \%$ of the sample $(n=28)$. Looking at the gray dashed line with triangles, this class is characterized by high endorsement of the coping strategies "Talked to Someone," "Avoidance," and "Problem Solving" and low endorsement of the coping strategies "Got mad" and "Worried/cried"; thus, we labeled this class the Adaptive Coping class and is the largest class, $63.8 \%$ of the sample $(n=283)$. The final class, comprising $31.1 \%$ of the sample $(n=183)$, is characterized by the low endorsement of all five coping items ("Talked to Someone," "Got Mad," "Avoidance," "Problem Solving," and "Worried/Cried"). This class shown by the light gray line with squares in the figure was labeled the No Coping class.

The process of assigning substantive labels to the emergent classes is an important part of the modeling process and involves studying the items and the patterns of response classes. Collectively, the pattern of endorsement within a given class and across classes is used to create labels. Creating labels for the classes deserves thoughtful consideration of the literature to try to provide meaningful names to describe the classes that accurately represent the emergent patterns. Often, the emergent classes present a complex solution and labeling classes requires careful consideration to create succinct labels that feel non-reductive. It is ultimately the responsibility of the researcher to create and justify the class labels, which involves referring back to the item probability plots in consideration of different class names.

\section{LCA: An Overview of the Statistical Model}

Using the coping example, we will describe the two key sets of parameters for the statistical expression of the LCA model, item endorsement probabilities, and class size proportions. In this coping example, we have five binary items, or the latent class indicators, $u_{1}, u_{2}, \ldots, u_{5}$ on a sample of $n=446$ youth. The LCA model assumes that the five items are measures of an underlying unordered (i.e., nominal) categorical latent class variable $C$, which has $K$ latent classes, where a modeling task is to decide on how many classes there are ( $K=$ How many?).

A specific class, denoted lowercase $k$, is represented by the exhaustive set of $K$ classes or latent groups. The relative size of each latent class, or the proportion of individuals in class $k, P(c=k)$, is denoted by $\hat{\pi}_{k}$. In the coping example, $\pi_{1}=.636$, which when converted to a percentage implies that $63.6 \%$ of the sample is in the Adaptive Coping class. It should be noted that the absolute class size or the number of observations $n_{k}$ should also be considered when making enumeration decisions. Even if the relative class size is small (e.g., $<5 \%$ ), if the overall sample size is sufficiently large, enumerating small classes may be stable and justifiable, given sufficient substantive support (Nylund-Gibson $\&$ Choi, 2018). For research contexts where low prevalence groups are of specific interest, as is often the case in psychology (e.g., highly aggressive youth, high substance abuse groups, etc.), a large sample would be needed to be able to identify and empirically estimate those classes.

Model assumptions. There are several assumptions that define the LCA model. The LCA model implies that the $K$ classes are mutually exclusive and exhaustive; each individual is assumed to be in one class and that the entire sample is assigned class membership. In a post hoc step, each observation is also given a posterior probability $p_{k}$ of being in each of the $K$ latent classes such that $\Sigma p_{k}=1$. This implies that any given observation can have partial membership in multiple classes.

The LCA model assumes that items are uncorrelated, given the latent class variable (i.e., no correlated disturbances) that is similar to the assumption in factor analysis. This assumption, the conditional independence assumption, implies that latent class membership explains all of the shared relationships among the observed items. This assumption can be relaxed to allow for within-class item relationships, above and beyond the latent class variable. Adding these within-class correlations can help prevent the 
over-extraction of latent classes (Masyn, 2013) because these relations, if ignored, can manifest in a signal for the need of more classes. Best practices about including these relations are still not established and are not commonly used.

LCA model parameters. As LCA is a multivariate modeling approach, the statistical expression of the model involves expressing the joint probability of the observed items. When expressing the probability of an individuals' response patterns, or response vectors, we discuss the probability of the joint expression of all items considered or the joint distribution of all the items. With respect to the latent class variable, $C$, under the conditional independence, the model is expressed as,

$$
\left.\operatorname{Pr}\left(u_{1 i}, u_{2 i}, \ldots, u_{M i}\right)=\sum_{k=1}^{K}\left[\pi_{k} \cdot\left(\prod_{m=1}^{M} \operatorname{Pr} u_{m i} \mid c_{i}=k\right)\right)\right] .
$$

Because binary or multicategorical items are used in LCA, the model uses logistic regression to capture the association between the observed indicator and the latent class variable. These logits are akin to a factor loading in factor analysis, in that they describe the relationship between the observed indicator and the latent variable. A logit value for each indicator is estimated for each latent class; thus, they are considered class-specific parameters. The conditional item probabilities are often parameterized as single thresholds (which is a negative logit) on the inverse logit scale as follows,

$$
\operatorname{Pr}\left(u_{m i}=1 \mid c_{i}=k\right)=\frac{1}{1+\exp \left(\tau_{m k}\right)},
$$

while the class proportions are parameterized as intercepts on the inverse multinomial scale such that:

$$
\pi_{k}=\operatorname{Pr}\left(\mid c_{i k}=k\right) \frac{\exp \left(\gamma_{o k}\right)}{\sum_{j=1}^{k} \exp \left(\gamma_{o j}\right)},
$$

where the intercept of the last class is set to zero, $\gamma_{o k}=0$, for identification.

The second set of parameters in the LCA model is the set of conditional item probability parameters, $\pi_{m k}$. These are the parameters that we use in the conditional item probability plot shown in Figure 1 and describe the LCA solution. The conditional item probabilities are the measurement parameters in the unconditional LCA model, whereas the relative class size parameters, $\gamma_{o k}$ are structural parameters and describe the relative sizes of the classes. Although it is possible to partially relax the conditional independence assumption, researchers should rely on a priori substantive knowledge and proceed with caution as to consider the sample-specific (and potentially idiosyncratic) nature of observed covariation that are ostensibly expressed as violations of this assumption. Other types of mixture models, some of which relax the conditional independence assumption, include growth mixture models (B. O. Muthén et al., 1999; Ram \& Grimm, 2009) and factor mixture models (Lubke \& Muthén, 2005).

\section{Class Enumeration: Deciding on the Number of Classes}

In this application, we used the five binary coping items (see Table 1) to fit a series of LCA models (1-5 latent classes), and in the previous section, we described the threeclass solution. In practice, class enumeration, the process of deciding on the number of classes, involves collecting fit information from all models, which are then used to compare models and decide on a final solution. What follows is a summary of the current best practices in class enumeration, followed by the enumeration process for the coping example used in this article.

One of the most time-consuming, although critical, parts of using mixture models is class enumeration. This is a critical step because the latent class variable becomes the focal variable of interest. Furthermore, after deciding on a solution, we become interested in associations between group membership in the latent classes and auxiliary variables (e.g., covariates and distal outcomes). Class enumeration involves fitting LCA models with a different number of classes to decide on how many classes best describe the patterns observed in the data. As in SEM, there is not a single fit index that is agreed upon for use in class enumeration, but rather, we use a set of fit indices to decide. These fit indices often do not all point to a single solution; it is recommended that we jointly consider statistical fit indices, substantive interpretability and utility, and classification diagnostics that help to illuminate how well the classes are differentiating the patterns (Masyn, 2013; B. O. Muthén, 2003).

The procedure starts with fitting a 1-class LCA model which is simply estimating the item endorsement probabilities (the observed item proportions) for the sample as a whole as there is only a single class. We then increase the number of classes, $k$, by 1 , examining whether the addition of each class results in conceptually meaningful and statistically better fitting solutions. We stop estimating additional classes when an increase in the number of classes does not result in a meaningful increase in model fit or convergence issues are encountered (e.g., due to identification problems or overparameterization). Convergence issues are indicated by error messages from the software package being used, often with details about the source of the problem. MplusAutomation greatly reduces the time required to implement this procedure by automating the process of 
estimating sets of models and summarizing them with tables and figures. We provide code on how to estimate models and tabulate a set of LCA models using MplusAutomation in Supplemental Material.

Fit indices used in LCA. There is no single fit index used in mixture models, although simulation studies provide guidance in identifying the correct number of classes in an LCA. In any given data analysis setting, however, we do not know the "true" number of classes, so it is the hope that these fit indices help us to approximate the correct number of classes, assuming that evidence of latent classes exists in a given population.

Information criterion. The information criteria (IC) are recommended for use in mixtures, specifically the Bayesian Information Criterion (BIC), Sample-size adjusted Bayesian Information Criterion (aBIC), Consistent Akaike Information Criterion (CAIC), and Approximate Weight of Evidence Criterion (AWE). These are all approximate fit indices where lower values indicate superior fit. For example, in a data analysis setting, we would compare the values of the BIC across the set of fitted models and would pick the model that has the lowest value of the BIC. It can be useful to plot the values of the BIC, ABIC, CAIC, and AWE to visually display the values and provide for easy inspection (Figure S1 in Supplemental Material). In practice, it is not uncommon that the BIC continues to decrease for each additional class added (i.e., there is not a global minimum) and in these instances, these plots can be particularly useful to inspect for an "elbow" or point of "diminishing returns" in model fit (e.g., small decreases in the IC for each additional latent class; Nylund, Bellmore, et al., 2007).

Likelihood-based tests. In addition, we consider likelihood-based tests. In mixture models, we do not use the standard chi-square difference test like we do in SEM (see Nylund, Asparouhov, \& Muthén, 2007) and instead use two alternatives: the Vuong-Lo-Mendell-Rubin adjusted likelihood ratio test (VLMR-LRT) and the bootstrapped likelihood ratio test (BLRT) — which provide $p$ values assessing whether adding a class leads to a statistically significant improvement in model fit. These likelihood-based tests compare the fit between two neighboring class models (e.g., a 2-class vs. a 3-class model), where a nonsignificant $p$ value for a $k$-class solution thus lends support for the $k-1$ class solution. See Nylund, Asparouhov, and Muthén (2007) for more details on ICs and likelihood-based tests and their utility in enumerating classes in mixture models.

Finally, two fit indices, borrowed from the Bayesian framework, have been used in the context of LCA, namely, the Bayes Factor $(B F)$ and the correct model probability $\left(c m P_{k}\right)$. While the $\mathrm{BF}$ is a pairwise comparison of fit between two models, the $c m P_{k}$ provides an estimate of each model being "correct" out of all models considered, assuming that the "true" model is indeed among them; the model with the largest value is selected. However, as each of these indices contains the BIC in the root of their formula, they are often highly correlated with BIC and thus provide partially redundant information.

Substantive consideration. When choosing the number of classes, researchers often consider the statistical evidence as well as the theoretical utility of each class among the set of competing models (B. O. Muthén, 2003). Following this logic, after enumeration has identified a reduced set of candidate models, theory, existing literature, and model utility are also considered. Here we are adopting a pragmatic perspective about the purpose of LCA in the exploratory context of class enumeration. For example, in the case of behavioral science research, the clinical or practical use of the latent classification acts as an important factor when deciding the number of classes for the final model. This reliance on theory necessitates the need for the replication of results in future studies (e.g., confirmatory LCA/latent profile analysis, LPA) to confirm that the emergent solution is substantiated in other studies (Schmiege et al., 2018).

\section{Demonstrating Class Enumeration With the Coping Example}

Returning to the coping example, we estimated 1 to 5 latent classes with Mplus (Muthén \& Muthén, 2017) and tabulated model fit using the MplusAutomation package in $\mathrm{R}$ (Hallquist \& Wiley, 2018), where the R code for this procedure can be found in Supplemental Material. Results, presented in Table 2, evidenced consecutive incremental worsening of fit in the 4-class and 5-class models. As is often the case, the collection of fit indices showed mixed endorsement between two competing models (i.e., the 2-class and 3-class models), and there is inconclusive support for a single model. The BIC, CAIC, VLMR-LRT, BF, and $\mathrm{cmP}_{k}$ (see Table 2 and Figure $\mathrm{S} 1$ in Supplemental Material) provide support for the 2-class solution, other indices, class-separation, and theoretical considerations suggest a 3-class model. Both the aBIC and the BLRT indices provide statistical support for the 3-class solution. Furthermore, upon a close comparison of the item probability plots for the 2- and 3-class models, the additional class provides a distinctive pattern of item endorsement that is missed using the 2-class model. The conditional item probability plot for the 2-class solution is presented in Figure 1 .

Because of the lack of agreement between the fit indices, theoretical and substantive factors were considered in the enumeration process (B. O. Muthén, 2003), which is always recommended to better understand alternative solutions. The theoretical relevance of the third class, along with the inconsistency of the fit indices, provides a strong rationale 
Table 2. Model Fit Indices for the LCA Model With I Through 5 Latent Classes.

\begin{tabular}{|c|c|c|c|c|c|c|c|c|c|c|}
\hline Model (k-class) & $L L$ & npar & CAIC & $\mathrm{BIC}$ & $\mathrm{aBIC}$ & AWE & VLMR-LRT $p$ value & BLRT $p$ value & $B F$ & $\mathrm{~cm} P_{k}$ \\
\hline I-class & $-1,294.0$ & 5 & $2,623.55$ & $2,618.55$ & $2,602.68$ & $2,664.05$ & & & & 0.0 \\
\hline 2-class & $-1,259.2$ & II & $2,596.58$ & $2,585.58$ & $2,550.67$ & $2,685.69$ & $<.001$ & $<.001$ & $>10$ & 1.0 \\
\hline 3-class & $-1,249.2$ & 17 & $2,619.01$ & $2,602.01$ & $2,548.06$ & $2,756.72$ & .125 & .004 & $>10$ & 0.0 \\
\hline 4-class & $-1,244.3$ & 23 & $2,651.84$ & $2,628.84$ & $2,555.85$ & $2,838.14$ & .012 & .263 & $>10$ & 0.0 \\
\hline 5-class & $-1,242.4$ & 29 & $2,690.63$ & $2,661.63$ & $2,569.60$ & $2,925.54$ & .352 & 1.000 & $>10$ & 0.0 \\
\hline
\end{tabular}

Note. Bold values indicate the model that the fit criteria endorse. $K=$ number of classes; $L L=$ log-likelihood; npar $=$ Parameters, $C A I C=C o n s i s t e n t$ Akaike Information Criterion; BIC = Bayesian Information Criterion; aBIC = adjusted BIC; AWE = Approximate Weight of Evidence Criterion; BLRT = bootstrapped likelihood ratio test; VLMR-LRT = Vuong-Lo-Mendell-Rubin adjusted likelihood ratio test; $p=p$ value; $B F=$ Bayes Factor; $\mathrm{cmP}=$ correct model probability. Bolded values indicate "best" fit for each respective statistic.

for endorsing this solution. Taken together, the 3-class solution was chosen as the final model in this example. Supporting this decision, previous research has identified an externalizing coping behavior class with low prevalence but clinical significance, showing convergence toward this model solution (Waasdorp \& Bradshaw, 2011). In the coping literature, engaging in externalizing behaviors to cope is typically considered to be maladaptive for youth and a focus for intervention (Modecki et al., 2017).

Challenges of enumeration. In practice, it is not uncommon that the statistical fit indices do not converge on one single model. This can occur for a variety of reasons: (a) there is not a single statistical index that performs equally across all mixture modeling contexts which leads to multiple plausible solutions, (b) sample size is inadequate to detect rare classes, (c) items are correlated after classification suggesting dimensions not well identified by the LCA, or (d) poor measurement quality blurs or multiplies "true" class distinctions.

In the case of multiple candidate models, we recommend that each solution be studied closely (Ram \& Grimm, 2009) by looking at the emergent classes, their profiles, and their relative size. Researchers should consider how the selected models relate to each other (e.g., is one an expanded version of the other?) and the stability of the different models. For example, when adding a class, which $k$-class prevalence changes, in comparison to the $k-1$ class? Is a class being split into two classes? This can be seen in our coping example in which the 2-class solution of the no coping class, made up $35.7 \%$ of the sample. In contrast, in the 3-class solution, the no coping class is split into a unique response pattern that was labeled the externalizing behavior class.

The enumeration decision should also include consideration of the relative sizes of the emergent classes. For example, are the emergent classes relatively large with respect to the proportion of the total sample, and does this represent a sufficient number of individuals to make up a stable solution? Simulation studies have shown that small or "rare" classes are generally difficult to recover in small samples and when class prevalence is highly unequal (Morovati,
2014; Tofighi \& Enders, 2008; Tueller \& Lubke, 2010). As such, we encourage researchers to be mindful of selecting an over extracted and potentially unstable class solution while also recognizing that there are instances where small or rare groups are of theoretical or practical importance.

Furthermore, it is often the case that small or low-frequency classes are of central interest in social sciences. For example, patterns such as heavy drug use or sexual assault have low prevalence but may be pertinent to research questions or interventions. Depending on sample size and power, a small class may not be identified in the context of mixture models because of the signal-to-noise ratio (Holt et al., 2017). Consequently, despite the challenge and tenability of results from rare classes, it is important to consider their real-world significance before dismissing them based solely on statistical considerations.

One potential limitation of the mixture modeling framework is that the blending or conflating of rare classes may be an artifact of inadequate sample size. This is an important and ongoing area of research, in which a researcher must strike a balance between epistemological truth and practical utility. For example, in highly complex systems such as human behavior, complexity may need to be distilled into simplified models with results that are accessible and meaningful to a broad audience. Therefore, the research goal may not necessarily be to discover the true class structure that perfectly characterizes the variation in a population but rather to make a fuzzy classification that provides a meaningful description of the data patterns for a particular purpose. Taking together these considerations should inform which final model is selected.

\section{Model Evaluation: Classification Quality}

Classification accuracy and precision are a way to evaluate LCA solutions after enumeration has been completed. The importance of classification will vary across the applied context of the LCA analysis. Several classification diagnostics tools exist to assess classification accuracy. These tools are not used in deciding on the number of classes but rather to understand the classification precision of the chosen 
Table 3. Model Classification Proportions and Accuracy.

\begin{tabular}{|c|c|c|c|c|c|c|c|}
\hline k-class solution & k-class & k-class proportions & $90 \% \mathrm{Cl}$ & mcaPk & AvePPk & OCCk & Entropy \\
\hline \multirow[t]{2}{*}{ 2-Class } & Class I & .65 & {$[.60, .7 I]$} & .611 & .924 & 6.55 & .531 \\
\hline & Class 2 & .35 & {$[.29, .40]$} & .389 & .783 & 6.70 & \\
\hline \multirow[t]{3}{*}{ 3-Class } & Class I & .64 & {$[.59, .70]$} & .597 & .852 & 3.19 & .716 \\
\hline & Class 2 & .05 & {$[.04, .08]$} & .059 & .926 & 228.50 & \\
\hline & Class 3 & .30 & {$[.26, .36]$} & .344 & .761 & 7.27 & \\
\hline
\end{tabular}

Note: mcaP $_{k}=$ Modal class assignment; $A_{v e P P_{k}}=$ Average posterior probability of correct classification; OCC $_{k}=$ Odds of correct classification ratio; $\mathrm{Cl}=$ confidence interval.

model. Classification quality assumes that the model is "correct," which may not be true and if incorrect would influence classification quality.

The average posterior probability of correct classification for each class $\left(A v e P P_{k}\right)$ is a classification metric that describes the proportion in each class that is correctly classified in each of the $k$-classes. Perfect classification would be indicated by an average posterior probability of 1 . In the Mplus model output, class-specific classification accuracy is found in the diagonal of the $A v e P P_{k}$ matrix with the offdiagonals corresponding to the probability of misclassification in the $k-1$ remaining classes. It has been suggested that $A v e P P_{k}$ values along the diagonal of the matrix that are greater than .7 indicate adequate classification precision for each specific class (Nagin, 2005).

The odds of correct classification ratio $\left(O C C_{k}\right)$ provide another measure of class-specific classification accuracy, which uses the model-estimated class proportion and the AvePP $P_{k}$ to show the odds of being in class- $k$ relative to classification based on random assignment. Therefore, larger values of $O C C_{k}$ indicate high classification accuracy (i.e., $O C C_{k}>5$; Nagin, 2005). The modal class assignment $\left(\right.$ mcaP $\left._{k}\right)$ is the portion of individuals modally assigned to class $k$ (e.g., if each observation is classified into one class). Finally, entropy $\left(E_{k}\right)$, which ranges between 0 and 1 (as implemented in $\mathrm{Mplus}$ ), provides a summary for the overall sample of classification precision of the model, where values $<.8$ are considered high (Clark \& Muthen, 2009; Ram \& Grimm, 2009). Classification of diagnostic information is useful to assess the performance of the selected model at classifying individuals. When considering the purpose or application of a model, classification accuracy and precision may be of primary importance. For example, if membership in a given class is used for diagnostic purposes or for treatment eligibility, it would be critical to pick a model with good classification performance across multiple measures of classification metrics.

The classification diagnostic information (Thibodeau et al., 2019) presented in Table 3 suggests that there is a moderately strong classification of individuals based on the 3 -class model solution. Starting from left in Table 3 the
" $k$-class proportion" $\hat{\pi}_{k}$ column represents the model estimated proportions for the sizes of the three classes, followed by the $90 \%$ bootstrapped confidence interval (CI) for that proportion. The column labeled " $m c a P_{k}$," the modal class assignment proportion, is the relative size of the class based on the modal class assignment. The differences between these two ways of estimating class proportionsthe model estimated $k$-class proportions and modal class assignment proportions - for any given class suggest classification error. Within the CIs, the modal class assignment proportion ideally should fall within the bounds of the corresponding $90 \%$ bootstrapped CI. For example, for Class 1 in Table 3, the mcaP $_{k}$ of .597 falls in the $90 \% \mathrm{CI}$, thus indicating there is minimal error in that class.

In the 3-class coping example, the average posterior probabilities, AvePP ${ }_{k}$, are all above the desired threshold of .7 and are thus considered adequate classification accuracy (Masyn, 2013). Of note, the smallest class, "Class 2," is well classified $\left(A v e P P_{k},=.926\right)$ which contributes to the stability of this solution and reduces the likelihood of classification bias. The odds of correct classification $\left(O C C_{k}\right)$ show that Classes 2 and 3 are well above the threshold criteria of 5 (Nagin, 2005), while Class 1 falls below the threshold with an odds of correct classification at just more than 3 to 1 . The modal assignment $\left(\mathrm{mcaP}_{k}\right)$ for each coping strategy class falls within the corresponding $90 \%$ CI suggesting adequate classification. And finally, the entropy $\left(E_{k}\right)$ is .72 which, in contrast to the other indices, is lower than ideal for overall model classification precision.

\section{Extension of LCA With Auxiliary Variables}

Applied research in the study of behavioral disorders may involve proposing complex questions about the interrelations between the emergent latent classes, the demographic makeup of the sample or other concurrently measured variables, and temporally distant outcomes, which are referred to as auxiliary variables. In the coping example, for example, we hypothesize that those who identify as experiencing a situation as being stressful (covariate) may have different 


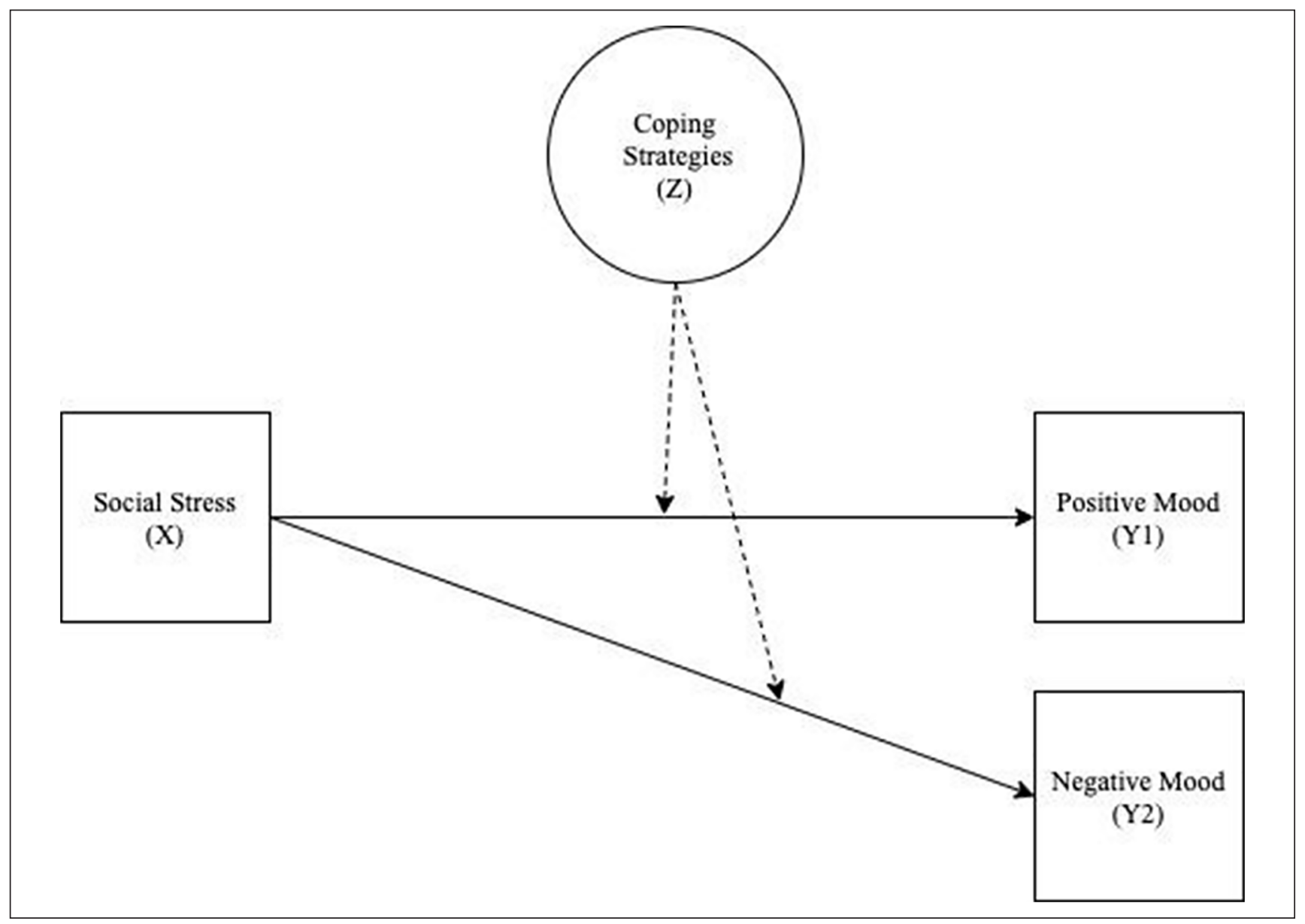

Figure 2. Path diagram of SEM model: The relationship between Social Stress and the outcome variables Negative Mood and Positive Mood are moderated by the latent class coping variable.

prevalence depending on their pattern of coping, measured by the latent class variable (moderator), or that coping patterns will predict positive or negative mood (distal outcome; see Figure 2).

Exploring the associations between a latent class variable, predictors, and outcome variables necessitates a method that accounts for the classification error and the associations with the auxiliary variables (Nylund-Gibson et al., 2014). Approaches have been developed to properly estimate auxiliary variable relations with the latent class variable, each of which uses multiple estimation steps to accommodate the latent class variable within a larger latent variable modeling framework (i.e., structural equation modeling; SEM). These multi-step procedures include the ML 3-step method (Vermunt, 2010), the BCH method (Bolck et al., 2004), and the two-step method (Bakk \& Kuha, 2018). All of these approaches accommodate multiple covariates and outcome variables, such as models that include (a) the latent class variable as covariate, (b) the latent class variable as a mediator, (c) the latent class variable as outcome variable, and (d) the latent class variable as moderator (see; McLarnon \& O’Neill, 2018). The BCH has outperformed the ML approach in simulation studies but has known issues with mixture models that have high classification error, namely, it estimates negative $\mathrm{BCH}$ weights making it unusable (Asparouhov \& Muthén, 2014). The 2-step procedure has outperformed the other multistep methods and is implemented in Latent Gold but not Mplus yet. For more on the comparison of methods see Bakk and Kuha (2021). For procedures to specify the ML 3-step in $\mathrm{R}$ with models estimated in Mplus using MplusAutomation (Hallquist \& Wiley, 2018) see Garber (2021) and Supplemental Material.

In the current article, we extend the inclusion of auxiliary variables to a context that includes both a covariate and a distal outcome. Specifically, the latent class variable (coping class) is specified as a moderating variable of the association between social stressor (e.g., feelings of rejection or experiencing conflicts with others) and subsequent mood (both positive and negative). In our example, the $\mathrm{BCH}$ method produced negative weights, so we use the ML 3-step manual method. 


\section{Incorporating Latent Class Variables in a Moderation Model}

Moderation analysis provides a context where we can study the effects of a predictor variable $(X)$ on an outcome variable $(Y)$, conditioned upon the level of the moderator $(Z)$. Within the latent variable framework, we consider the special case when the moderator is the latent class variable that is uniquely specified in mixture modeling contexts. Specifically, with an LCA variable as a moderator (see Figure 2), we allow the relationship between $X$ and $Y$ to be estimated for each class. This is specified by including $X \rightarrow Y$ regressions for each of the classes, thus allowing the regression parameters to vary by the latent classes (O'Neill et al., 2016). For example, if class membership represents profiles of coping patterns, these patterns can be theorized to moderate the relationship between the covariate and outcome. This variation in slope and intercept across classes can be visually reported using a traditional simple slopes graph.

Evidence for moderation is found in the class-specific estimates and is demonstrated in the coping example. To test for the significance of evidence of class differences in slope and intercepts, omnibus Wald tests can be conducted (see Supplemental Material). Testing for post hoc pairwise intercept and slope differences can also be specified individually. Intercept differences represent how the distal outcome variable varies by class membership. Significant slope differences $(\beta)$ indicate moderation.

\section{Heterogeneity in Coping: The Applied Example}

To illustrate the modeling process, we used data collected on coping strategies that adolescents use in response to everyday stressors. We hypothesized that there are patterns in how these youth use the set of coping skills, so we used LCA to explore which patterns emerged. The coping measures used in this study were based on work conducted by Causey and Dubow (1992) assessing the scale validity of five coping strategies that fall along two dimensions, approach and avoidance. Approach strategies include seeking social support ("Talked to Someone") and problemsolving ("Problem Solving"). Avoidance strategies involve distancing ("Avoidance"), internalizing ("Worried/Cried"), and externalizing ("Got Mad") behaviors. These coping strategies were developed to capture the range of responses relevant to the social and academic stressors children and adolescents face. Most scholarship using coping measures assesses each coping strategy individually and examines associations between individual strategies and adjustment indices (e.g., Kingsbury et al., 2016), rather than propensities to use several strategies together (e.g., Waasdorp \& Bradshaw, 2011) as considered here. Examination of patterns is better suited to capturing how the specifics of situations may inform the strategies adopted rather than assuming individuals use and endorse one typical response across situations (Bellmore et al., 2013). In this example, we examine whether coping patterns moderate the association between social stress and mood.

\section{Moderation}

We first present the results of distal outcome means and their variation across the emergent latent class, then the results of the moderation analysis, which involves interpreting the mean slope difference across the class. As the moderator is the latent class variable $(Z)$, which has three coping classes, the moderation is exploring how students' moods ( $Y$; both positive and negative) are related to whether social stress is present or not $(X)$.

Distal outcome differences. Significant pairwise mean differences were tested across the classes for the outcome variables of positive and negative mood. The omnibus Wald test indicated that there were statistically significant relations with the outcomes (positive and negative mood) and the latent class variable, $\chi^{2}(2)_{p o s}=18.26, p<.001$; $\chi^{2}(2)_{\text {neg }}=7.24, p=.025$, which implies there is support to look at pairwise differences. In Figure 3, we see that students in the normative Adaptive Coping class, representing $64 \%$ of the sample, had the highest average positive mood scores $(M=3.16)$. In contrast, students in the Externalizing Behavior class had, on average, lower positive mood $(M=2.67)$. The No Coping class had a similar, moderately low, average positive mood $(M=2.72)$. Pairwise tests of distal means for positive mood found significant differences between the Adaptive Coping class and both the Externalizing Behavior class and the No Coping class. Similarly, pairwise tests of distal means for negative mood found significant differences between the Externalizing Behavior class and both the Adaptive Coping class and the No Coping class. Those students who were distinguished from other students by their endorsement of using externalizing coping strategies reported higher levels of negative mood than did students who used adaptive coping or minimal coping strategies. All other pairwise differences were not significant at the $\alpha=.05$.

\section{Moderation Model: Slope Differences}

A model with the latent class coping variable as moderator was specified to model the associations between the predictor $(X)$ social stress and the outcome $(Y)$ mood. Coping class membership is hypothesized to moderate the relationship of $X \rightarrow Y$. Separate moderated logistic regressions $\left(X_{k} \rightarrow Y_{k}\right)$ are estimated for each level of the latent coping class variable, so regression intercepts and slopes are estimated for each class. Regression coefficient slope differences across coping classes indicate an interaction effect 


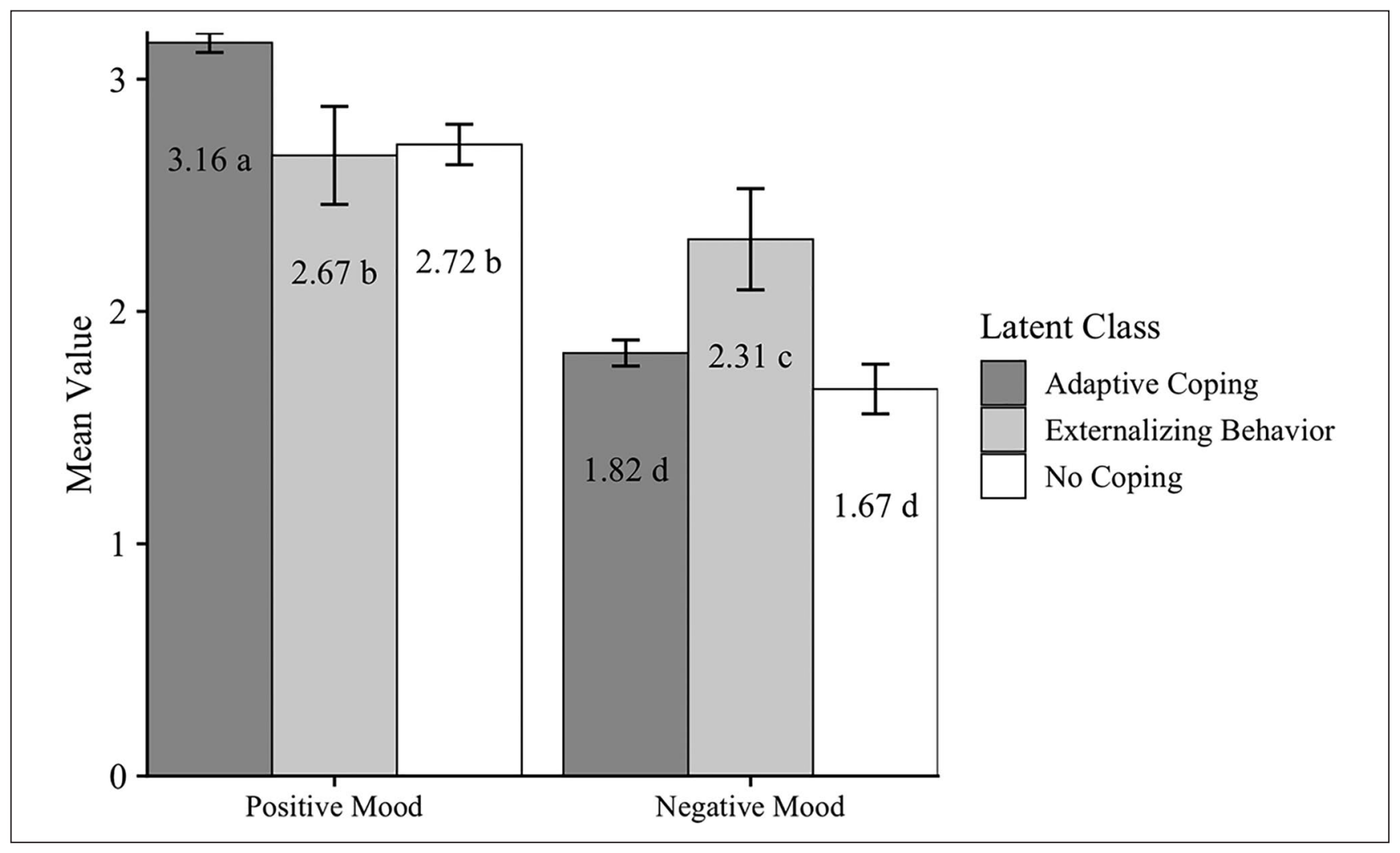

Figure 3. Distal outcomes for positive and negative mood mean differences.

Note. Values followed by subscripts with the same letter refer to nonsignificant pairwise mean differences. Values with different subscripts represent significant pairwise mean differences.

implying a conditional relationship between variables, social stress, and mood based on group membership. To test for significance of pairwise slope differences, Wald tests were conducted for each outcome. Omnibus tests showed that coping moderated the relation between social stress and negative mood but did not moderate the relation between social stress and positive mood, $\chi^{2}(2)_{\text {negative }}=9.59$, $p=.008 ; \chi^{2}(2)_{\text {positive }}=4.06, p=.131$, respectively.

Positive mood. For the outcome variable positive mood, evidence of moderation by the latent variable coping strategies was not found. Although the regression of positive mood on social stress was significant for the Adaptive Coping class, $\beta=0.31,95 \%$ CI $[0.16,0.47], p<.001$, the coefficients for the Externalizing Behavior class and No Coping class were nonsignificant, indicating that these slopes were not significantly different than zero (see Figure 4 and Table S3 in Supplemental Material). Despite the significant slope of the Adaptive Class, examination of the pairwise slope differences for positive mood found no significant differences.

Negative mood. In examination of the simple slopes for the outcome variable negative mood, evidence of moderation by the latent variable coping strategies was found. For the Externalizing Behavior class, the regression of negative mood on social stress was found to be significant, $\beta=0.73$, $95 \%$ CI $[0.07,1.59], p=.04$ (see Figure 4 and Table S3 in Supplemental Material). This result indicates that for students in the Externalizing Behavior class, the mean negative mood is higher for those who report that the event was a social stressor. For the Adaptive Coping class, the regression of negative mood on social stress was negative and significant, $\beta=-0.41,95 \%$ CI $[-0.62,-0.25], p<.001$, suggesting that for students in the Adaptive Coping class, negative mood was slightly lower when the event involved social stress. For the No Coping class, the regression for negative mood on social stress was nonsignificant which indicates that the mean negative mood was not significantly different for students in this class who had reported a social stress and those who did not.

The examination of the pairwise slope differences for negative mood regressed on social stress, the Externalizing Behavior class was found to vary significantly from the Adaptive Coping class, Wald $\chi^{2}(1)=.49, p=.033$, and $N o$ Coping class, Wald $\chi^{2}(1)=.65, p=.007$ (see Figure 4). Slope differences between the Adaptive Coping class and No Coping class did not reach significance. 


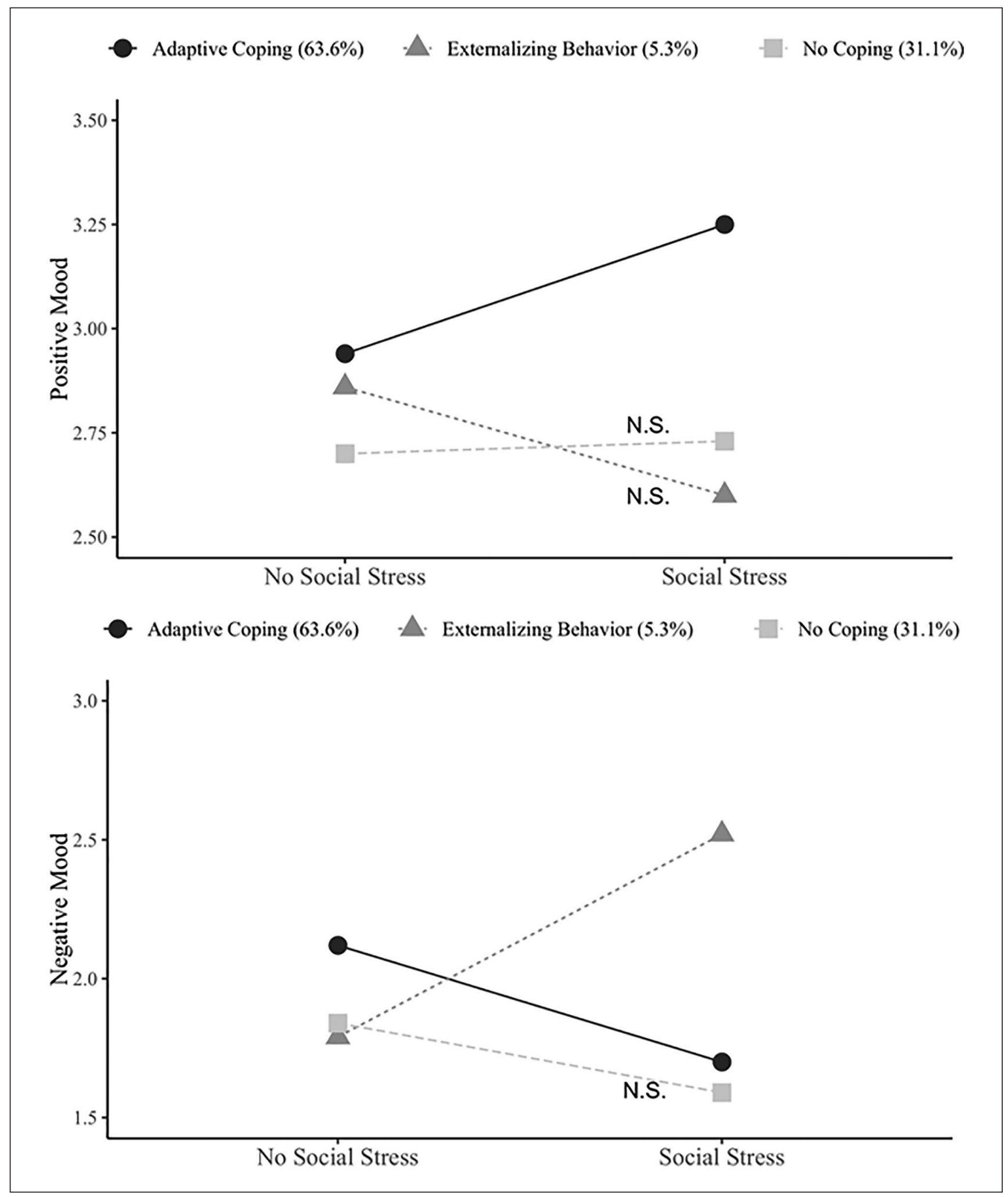

Figure 4. Moderation of positive/negative mood and social stress by the three coping latent classes. Note. N.S. = slope coefficient is not significantly different from zero. 


\section{Discussion}

In this article, we present the latent class model in a pedagogical fashion to help facilitate understanding of its use in applied work. We extended the LCA model by embedding it in a larger modeling context, specifically in a moderation context. Doing this opens up a modeling perspective less widely seen in the applied literature. By demonstrating the use of the latent class variable as a moderator in a simple regression model, we hope that applied researchers will utilize this model to enable more nuanced theories about population heterogeneity.

In addition, we provide heavily annotated $\mathrm{R}$ code for all models considered in this article utilizing MplusAutomation (Hallquist \& Wiley, 2018). This software provides an efficient manner to estimate batches of models and organize file scripts in a self-contained location. We provide all the codes for these models in Supplemental Material that can serve as a valuable resource to those learning latent class or latent class moderation.

The results of the coping example highlight the utility of examining patterns of coping responses as moderators of the stress-mood association. Importantly, the patterns for coping that we found illustrate that it is incorrect to presume certain responses co-occur together because they fall along the same dimension. We did not find that approach responses uniformly co-occur with other approach responses within individuals. Rather, we found a class of individuals that uses both avoidance and approach (specifically "Problem Solving" and "Talking to Someone") in response to stressors. Examination of how coping classes moderate the association between social stress and mood takes our understanding of coping a step further by showing that the class that uses both avoidance and approach responses reports better adjustment than the other classes. This finding would not be evident if we took a factor analytic approach, sometimes called a variable approach, to examine moderation of social stress and mood even if we found that talking to someone, avoidance, and problem-solving independently moderated the association between social stress and mood.

The no-response group that we identified is also important to mention. Its existence is consistent with other studies showing a group of adolescents who do not endorse any of the coping options (Bellmore et al., 2013). It is only through the moderation analyses that we can understand whether no coping is more maladaptive than other approaches. In fact, we find that the students who do not endorse any coping responses report no difference in their positive or negative moods as a function of social stress. This is important as it demonstrates non-endorsement as distinct from an avoidance coping response. Finally, analyses indicated that the externalizing coping class, while expected to be maladaptive, was particularly so when considering a negative mood when the stressor was social in nature (i.e., involved another student). This finding shows how the context of the stressor may matter in determining the degree to which coping strategies serve to minimize or exacerbate poor outcomes.

No study is without limitations. As with many LCAs, our current analysis was limited in the ability to detect low prevalence classes. It is conceivable that among individuals with behavioral disorders there is a small subset with a pattern of coping strategies that either put them at risk or act as a protective factor. In either case, this low prevalence class is of interest to researchers. The sample size of the current study makes it difficult to investigate research questions about low prevalence groups. The smallest class enumerated in this study was the Externalizing Behavior class at $5.2 \%$ describing approximately 26 individuals. This small class cannot be interpreted confidently without replication and further validation. It is possible that the coping pattern found for this group is an artifact of the particular sample and would not generalize to the larger population or that they did not actually perceive the social stress as stressful. We used the ML 3-step procedure and did not explore for differences in item functioning across levels of predictors (c.f., Masyn, 2017), although future applications could incorporate that into the analysis before specifying the moderation model. Furthermore, the use of the 3-step with a sample of 446 may be underpowered (Collier \& Leite, 2017). Finally, in the current article, we do not adjust $p$ values for the multiple comparisons as there are no best practices and clear procedures developed for this in the mixture modeling context but is something that should be considered in future applications.

\section{Future Directions}

LCA models provide a modeling context that allows researchers to explore how multiple categorical variables interact to explore groups not directly observable. The mixture modeling framework features a number of advantages relative to alternative approaches to creating groups, providing a context where the error in group formation can be estimated together with other relations of interest (i.e., auxiliary variables). In this study, the applied example contains only a single latent class variable, but there are contexts where multiple latent class variables are applicable, either cross-sectional (e.g., coping and behavioral actions) or longitudinal (e.g., coping at two time points). With multiple latent class variables, it is possible to model the patterns among the latent classes across the two variables using the latent transition analysis model (i.e., LTA; Nylund, Bellmore, et al., 2007).

Another important decision related to enumeration is the choice of items or variable selection. Variables selected from a scale validated using conventional methods (e.g., factor analyses) may not be the best way to utilize mixture 
models because of the high correlation among items. This is a large and complex topic that has only recently begun to be explored (Fop \& Murphy, 2018). In addition, it could be that the LCA model is not the right model or the model is misspecified. One such example is when there are unspecified within class item correlations. More research is needed in this area and would be useful in providing recommendations on when this is the case.

We hope that this article provides an overview of research contexts in which LCA and moderation have utility in addressing important questions in the behavioral sciences. In addition, this study provides implication procedures to embed latent class models in a more general SEM context. In this study, we demonstrate a model with the latent class variable as a moderator by specifying a simple regression within each of the latent classes. Many other combinations of class-specific variable relations and parameter restrictions can be specified, opening the possibility of a wide range of other modeling ideas. The flexibility of the mixture modeling framework supports the exploration of a wide range of research questions.

\section{Declaration of Conflicting Interests}

The author(s) declared no potential conflicts of interest with respect to the research, authorship, and/or publication of this article.

\section{Funding}

The author(s) disclosed receipt of the following financial support for the research, authorship, and/or publication of this article: The research reported in this article was partially supported in part by the Institute of Education Sciences, U.S. Department of Education, through Grant \# R305A170559 to the University of California, Davis and through Grant \# R305A160157 to the University of California, Santa Barbara. The opinions expressed are those of the authors and do not represent views of the Institute of Education Sciences or the U.S. Department of Education.

\section{ORCID iD}

Karen Nylund-Gibson iD https://orcid.org/0000-0003-3499-1052

\section{Supplemental Material}

Supplementary material for this article is available on the Behavioral Disorders website at https://journals.sagepub.com/ home/bhd.

\section{References}

Asparouhov, T., \& Muthén, B. (2014). Auxiliary variables in mixture modeling: Three-step approaches using Mplus. Structural Equation Modeling, 21(3), 329-341. https://doi.org/10.1080/ 10705511.2014 .915181

Bakk, Z., \& Kuha, J. (2018). Two-step estimation of models between latent classes and external variables. Psychometrika, 83(4), 871-892.
Bakk, Z., \& Kuha, J. (2021). Relating latent class membership to external variables: An overview. British Journal of Mathematical and Statistical Psychology, 74(2), 340-362.

Bellmore, A., Chen, W. T., \& Rischall, E. (2013). The reasons behind early adolescents' responses to peer victimization. Journal of Youth and Adolescence, 42(2), 275-284. https:// doi.org/10.1007/s10964-012-9825-0

Bolck, A., Croon, M., \& Hagenaars, J. (2004). Estimating latent structure models with categorical variables: One-step versus three-step estimators. Political Analysis, 12(1), 3-27.

Brusco, M. J., Shireman, E., \& Steinley, D. (2017). A comparison of latent class, $\mathrm{K}$-means, and $\mathrm{K}$-median methods for clustering dichotomous data. Psychological Methods, 22(3), 563-580. https://doi.org/10.1037/met0000095

Causey, D. L., \& Dubow, E. F. (1992). Development of a selfreport coping measure for elementary school children. Journal of Clinical Child and Adolescent Psychology, 21(1), 47-59. https://doi.org/10.1207/s15374424jccp2101_8

Clark, S. L., \& Muthén, B. (2009). Relating latent class analysis results to variables not included in the analysis.

Collier, Z. K., \& Leite, W. L. (2017). A comparison of three-step approaches for auxiliary variables in latent class and latent profile analysis. Structural Equation Modeling, 24(6), 819-830.

Dwyer, C. A. (1996). Cut scores and testing: Statistics, judgment, truth, and error. Psychological Assessment, 8(4), 360-362. https://doi.org/10.1037/1040-3590.8.4.360

Fop, M., \& Murphy, T. B. (2018). Variable selection methods for model-based clustering. Statistics Surveys, 12, 18-65. https:// doi.org/10.1214/18-ss119

Gage, N. A., Scott, T., Hirn, R., \& MacSuga-Gage, A. S. (2018). The relationship between teachers' implementation of classroom management practices and student behavior in elementary school. Behavioral Disorders, 43(2), 302-315. https:// doi.org/10.1177/0198742917714809

Garber, A. C. (2021). 3-step ML auxiliary variable integration using MplusAutomation. https://doi.org/10.31234/osf.io/phtxa

Hallquist, M. N., \& Wiley, J. F. (2018). MplusAutomation: An R package for facilitating large-scale latent variable analyses in Mplus. Structural Equation Modeling, 25(4), 621-638.

Holt, M. K., Felix, E., Grimm, R., Nylund-Gibson, K., Green, J. G., Poteat, V. P., \& Zhang, C. (2017). A latent class analysis of past victimization exposures as predictors of college mental health. Psychology of Violence, 7(4), 521-532. https://doi. org $/ 0.1037 /$ vio0000068

Kavčič, T., Avsec, A., \& Kocjan, G. Z. (2021). Coping profiles and their association with psychological functioning: A latent profile analysis of coping strategies during the COVID19 pandemic. Personality and Individual Differences, 185, Article 111287.

Kingsbury, M., Liu, J., Coplan, R. J., Chen, X., \& Li, D. (2016). Assessment and implications of coping styles in response to a social stressor among early adolescents in China. The. Journal of Early Adolescence, 36(2), 222-250.

Leon-Perez, J. M., Notelaers, G., Arenas, A., Munduate, L., \& Medina, F. J. (2014). Identifying victims of workplace bullying by integrating traditional estimation approaches into a latent class cluster model. Journal of Interpersonal Violence, 29(7), 1155-1177. 
Little, T. D., Cunningham, W. A., Shahar, G., \& Widaman, K. F. (2002). To parcel or not to parcel: Exploring the question, weighing the merits. Structural Equation Modeling, 9(2), 151-173.

Lubke, G. H., \& Muthén, B. (2005). Investigating population heterogeneity with factor mixture models. Psychological Methods, 10(1), 21-39.

Magidson, J., \& Vermunt, J. (2002). Latent class models for clustering: A comparison with K-means. Canadian Journal of Marketing Research, 20(1), 36-43.

Masyn, K. E. (2013). Latent class analysis and finite mixture modeling. In T. D. Little (Ed.), The Oxford handbook of quantitative methods (Vol. 2: Statistical analysis, pp. 551-611). Oxford University Press.

Masyn, K. E. (2017). Measurement invariance and differential item functioning in latent class analysis with stepwise multiple indicator multiple cause modeling. Structural Equation Modeling, 24(2), 180-197.

McLarnon, M. J., \& O’Neill, T. A. (2018). Extensions of auxiliary variable approaches for the investigation of mediation, moderation, and conditional effects in mixture models. Organizational Research Methods, 21(4), 955-982.

Modecki, K. L., Zimmer-Gembeck, M. J., \& Guerra, N. (2017). Emotion regulation, coping, and decision making: Three linked skills for preventing externalizing problems in adolescence. Child Development, 88(2), 417-426. https://doi. org/10.1111/cdev.12734

Morovati, D. (2014). The intersection of sample size, number of indicators, and class enumeration in LCA: A Monte Carlo study [Doctoral dissertation, UC Santa Barbara].

Muthén, B. O. (2003). Statistical and substantive checking in growth mixture modeling: Comment on Bauer and Curran (2003). Psychological Methods, 8(3), 369-377.

Muthén, B. O., Shedden, K., \& Spisic, D. (1999). General latent variable mixture modelling (Technical report). Santa Monica, CA: Muthén \& Muthén.

Muthén, L. K., \& Muthén, B. O. (1998-2017). Mplus user's guide (8th ed.).

Nagin, D. S. (2005). Group-based modeling of development. Harvard University Press.

Nylund, K., Bellmore, A., Nishina, A., \& Graham, S. (2007). Subtypes, severity, and structural stability of peer victimization: What does latent class analysis say? Child Development, 78, 1706-1722. https://doi.org/10.1111/j.14678624.2007.01097.x

Nylund, K. L., Asparouhov, T., \& Muthén, B. O. (2007). Deciding on the number of classes in latent class analysis and growth mixture modeling: A Monte Carlo simulation study. Structural Equation Modeling, 14(4), 535-569.

Nylund-Gibson, K., \& Choi, A. Y. (2018). Ten frequently asked questions about latent class analysis. Translational Issues in Psychological Science, 4(4), 440-461.

Nylund-Gibson, K., Grimm, R., Quirk, M., \& Furlong, M. (2014). A latent transition mixture model using the three-step specification. Structural Equation Modeling, 21(3), 439454. https://doi.org/10.1080/10705511.2014.915375

Nylund-Gibson, K., Grimm, R. P., \& Masyn, K. E. (2019). Prediction from latent classes: A demonstration of different approaches to include distal outcomes in mixture models. Structural Equation Modeling, 26(6), 967-985.

O’Neill, T. A., McLarnon, M. J., Xiu, L., \& Law, S. J. (2016). Core self-evaluations, perceptions of group potency, and job performance: The moderating role of individualism and collectivism cultural profiles. Journal of Occupational and Organizational Psychology, 89(3), 447-473. https://doi. org/10.1111/joop. 12135

Ram, N., \& Grimm, K. J. (2009). Methods and measures: Growth mixture modeling: A method for identifying differences in longitudinal change among unobserved groups. International Journal of Behavioral Development, 33(6), 565-576.

Schmiege, S. J., Masyn, K. E., \& Bryan, A. D. (2018). Confirmatory latent class analysis: Illustrations of empirically driven and theoretically driven model constraints. Organizational Research Methods, 21(4), 983-1001.

Thibodeau, E. L., Masyn, K. E., Rogosch, F. A., \& Cicchetti, D. (2019). Child maltreatment, adaptive functioning, and polygenic risk: A structural equation mixture model. Development and Psychopathology, 31(2), 443-456.

Tofighi, D., \& Enders, C. K. (2008). The impact of misspecifying class-specific residual variances in growth mixture models. Structural Equation Modeling, 15(1), 75-95.

Tsiantis, A. C. J., Beratis, I. N., Syngelaki, E. M., Stefanakou, A., Asimopoulos, C., Sideridis, G. D., \& Tsiantis, J. (2013). The effects of a clinical prevention program on bullying, victimization, and attitudes toward school of elementary school students. Behavioral Disorders, 38(4), 243-257. https://doi. org/10.1177/019874291303800406

Tueller, S., \& Lubke, G. (2010). Evaluation of structural equation mixture models: Parameter estimates and correct class assignment. Structural Equation Modeling, 17(2), 165-192.

Vermunt, J. K. (2010). Latent class modeling with covariates: Two improved three-step approaches. Political Analysis, 450-469. https://doi.org/10.1093/pan/mpq025

Vermunt, J. K. (2011). K-means may perform as well as mixture model clustering but may also be much worse: Comment. https://doi.org/10.1037/a0020144

Waasdorp, T. E., \& Bradshaw, C. P. (2011). Examining student responses to frequent bullying: A latent class approach. Journal of Educational Psychology, 103(2), 336-352.

Wang, Y., \& Yip, T. (2020). Sleep facilitates coping: Moderated mediation of daily sleep, ethnic/racial discrimination, stress responses, and adolescent well-being. Child Development, 91, e833-e852. https://doi.org/10.1111/cdev.13324

Whittaker, T. A., \& Miller, J. E. (2021). Exploring the enumeration accuracy of cross-validation indices in latent class analysis. Structural Equation Modeling: A Multidisciplinary Journal, 28(3), 376-390. 